\title{
Additions to the Human Plasma Proteome via a Tandem MARS Depletion iTRAQ-Based Workflow
}

\author{
Zhiyun Cao, ${ }^{1}$ Sachin Yende, ${ }^{2}$ John A. Kellum, ${ }^{2}$ and Renã A. S. Robinson ${ }^{1}$ \\ ${ }^{1}$ Department of Chemistry, University of Pittsburgh, Pittsburgh, PA 15260, USA \\ ${ }^{2}$ The Clinical Research, Investigation, and Systems Modeling of Acute Illness (CRISMA) Laboratory Center and Department of \\ Critical Care Medicine, University of Pittsburgh, Pittsburgh, PA 15260, USA
}

Correspondence should be addressed to Renã A. S. Robinson; rena@pitt.edu

Received 5 October 2012; Accepted 10 January 2013

Academic Editor: Visith Thongboonkerd

Copyright (C) 2013 Zhiyun Cao et al. This is an open access article distributed under the Creative Commons Attribution License, which permits unrestricted use, distribution, and reproduction in any medium, provided the original work is properly cited.

\begin{abstract}
Robust platforms for determining differentially expressed proteins in biomarker and discovery studies using human plasma are of great interest. While increased depth in proteome coverage is desirable, it is associated with costs of experimental time due to necessary sample fractionation. We evaluated a robust quantitative proteomics workflow for its ability (1) to provide increased depth in plasma proteome coverage and (2) to give statistical insight useful for establishing differentially expressed plasma proteins. The workflow involves dual-stage immunodepletion on a multiple affinity removal system (MARS) column, iTRAQ tagging, offline strong-cation exchange chromatography, and liquid chromatography tandem mass spectrometry (LC-MS/MS). Independent workflow experiments were performed in triplicate on four plasma samples tagged with iTRAQ 4-plex reagents. After stringent criteria were applied to database searched results, 689 proteins with at least two spectral counts (SC) were identified. Depth in proteome coverage was assessed by comparison to the 2010 Human Plasma Proteome Reference Database in which our studies reveal 399 additional proteins which have not been previously reported. Additionally, we report on the technical variation of this quantitative workflow which ranges from \pm 11 to $30 \%$.
\end{abstract}

\section{Introduction}

Discovery studies using plasma proteomics present challenges due to the technical difficulties associated with measuring the large dynamic range ( $10-12$ orders of magnitude) of proteins that exist in this medium [1]. Low-abundance proteins, which are of interest for biomarker applications, are often only accessible with involved proteomics workflows that utilize multiple sample fractionation steps. While the development of specific clinical immunoassays would resolve this approach, much work needs to be done in this area. Enrichment strategies for low-abundance plasma proteins rely on immunodepletion of high-abundance proteins [2-5], and, more recently, tandem depletion strategies have been employed [6-9]. For example, proteins present in as little as $1-1.6 \mu \mathrm{g} \cdot \mathrm{mL}^{-1}$ concentrations are detectable using tandem removal of abundant proteins with human serum albumin and Human 14 (Hu 14) multiple affinity removal system (MARS) columns [9]. A two-stage depletion setup that involves serial IgY and Supermix columns has also been effective in increasing the number of detectable low abundance proteins without affecting quantitative accuracy and precision using isobaric tags for relative and absolute quantification (iTRAQ) [6].

Recently, an updated reference database of human plasma proteins was released from the Human Proteome Organization which includes 1929 nonredundant protein sequences [10]. This list includes proteins that were identified amongst 30 laboratories that utilized various enrichment and depletion strategies, shotgun proteomics techniques, and liquid, chromatography tandem mass spectrometry (LC-MS/MS) platforms. Herein, we report additions to the released reference database based on results obtained from the analysis of plasma samples in our laboratory analyzed by a dual depletion shotgun proteomics technique.

Quantitative proteomics analyses of plasma are useful for identifying clinically relevant biomarkers [4] or in understanding disease mechanisms such as Alzheimer's disease [11]. 
The inherent biological variability across human patients can require a large number of samples in order to determine differentially expressed proteins that are statistically relevant. Depending on factors such as instrumental platform and available instrument time, multiplexing strategies are attractive. The commercial iTRAQ reagent allows up to eight samples to be multiplexed and has been effective in identifying biomarkers or differentially expressed proteins in diseases [12-14]. Limitations to this quantitative approach can include cost of reagent kits and issues with underestimation of ratios [15].

Nonetheless, iTRAQ can provide reliable quantitative information depending on the statistical rigor required for denoting proteins as differentially expressed [6, 16-19]. Several reports have stressed the importance of biological and technical replication in iTRAQ-based quantitative studies $[13,16,19,20]$. These reports, however, do not converge on the same finite set of criteria for determining statistically relevant differentially expressed proteins. For example, Song et al. suggest that at least 20 or 8 biological samples are required in order to use fold change cutoff values of 1.5 and 2.0, respectively [19]. Chee et al. employ a $\pm 30 \%$ or $\pm 50 \%$ cutoff for technical and biological replicates, respectively [16]. Most recently, a fold change $>2$ was deemed appropriate when at least six biological sample replicates are employed in order to have sufficient statistical power [21]. That the criteria should even converge has also been questioned as it has been proposed that fold change cutoff values are dependent on many factors: replications, number of observed peptides, protein class (e.g., high or low abundance), and so forth; specific values should be defined based on experimental goals and design [22].

Herein, we evaluate a robust tandem depletion quantitative proteomics workflow for its ability to provide additional insight to the human plasma proteome and to provide suitable criteria for the statistically relevant determination of differentially expressed proteins in human plasma.

\section{Materials and Methods}

2.1. Plasma Samples. Four plasma samples were obtained from patients enrolled in the Genetic and Inflammatory Markers of Sepsis (GenIMS) study [23]. These patients were initially diagnosed with community-acquired pneumonia upon admittance to the emergency department, and samples were collected; however, further diagnoses revealed improper initial assessment. Thus these samples come from otherwise healthy volunteers. Approval for the participation of human subjects was obtained by the institutional review board of the University of Pittsburgh and other participating sites.

2.2. Tandem MARS Immunodepletion (TMD). The $\mathrm{Hu} 6$ MARS column depletes serum albumin, IgG, $\alpha 1$-antitrypsin, IgA, transferrin, and haptoglobin proteins. An injection amount of $60 \mu \mathrm{L}$ of crude plasma was applied to the MARS column (Agilent; Santa Clara, CA, USA), and after the initial depletion, flow-through fractions were concentrated with a $5 \mathrm{~K}$ molecular weight cutoff concentrator (Agilent; Santa Clara, CA, USA) at $4695 \mathrm{~g}$ for 1.5 hours. Samples (hereafter referred to as $\mathrm{MD}$ ) were then stored at $-80^{\circ} \mathrm{C}$ or reinjected onto the MARS column for tandem MARS depletion. The second flow-through fractions (hereafter referred to as TMD) were concentrated, and protein concentrations were measured using the BCA protein assay.

2.3. Protein Digestion and iTRAQ Labeling. In order to normalize experimental conditions, similar amounts of protein (i.e., $100 \mu \mathrm{g}$ ) as determined from a BCA assay were employed. Protein amounts as opposed to sample volumes were used since the concentrations of proteins in the flow-through fraction may vary across samples after TMD. A total of $100 \mu \mathrm{g}$ of protein was denatured with an extraction buffer

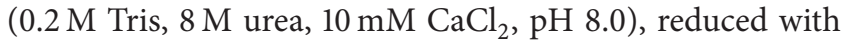
1:40 molar excess of dithiothreitol for $2 \mathrm{~h}$ at $37^{\circ} \mathrm{C}$, and then alkylated with 1:80 molar excess of iodoacetamide for $2 \mathrm{~h}$ on ice in the dark. The alkylation reaction was quenched by adding 1:40 molar excess of cysteine, and the mixture was incubated at room temperature for $30 \mathrm{~min}$. Molar excesses for each reagent was calculated based on an estimation of the total moles of protein in each sample (i.e., average $\mathrm{MW}$ of $\sim 66 \mathrm{kDa})$. Tris buffer $\left(0.2 \mathrm{M}\right.$ Tris, $\left.10 \mathrm{mM} \mathrm{CaCl}_{2}, \mathrm{pH} 8.0\right)$ was added to dilute the urea concentration to $2 \mathrm{M}$. Each sample was incubated with bovine TPCK-heated trypsin at $50: 1$ substrate: enzyme mass ratio for $24 \mathrm{~h}$ at $37^{\circ} \mathrm{C}$. Digested samples were desalted with an HLB cartridge (Waters; Milford, MA, USA) and dried by centrifugal evaporation. Each sample was labeled with an iTRAQ reagent following the manufacturer's protocol (Applied Biosystems; Foster City, CA, USA) with slight modifications. Briefly, each iTRAQ reagent was solubilized with $70 \mu \mathrm{L}$ ethanol and transferred to peptide mixtures. After $1.5 \mathrm{~h}$ of incubation, the reaction was quenched by adding $50 \mu \mathrm{L}$ of water. Labeled samples were mixed in $1: 1: 1: 1$ ratios for $i$ TRAQ reagents that generate reporter ions $m / z 114: 115: 116: 117$, respectively.

2.4. Offline SCX Fractionation. For strong-cation exchange (SCX) liquid chromatography the separation was carried out on a polysulfoethyl $100 \mathrm{~mm} \times 2.1 \mathrm{~mm}, 5 \mu \mathrm{m}, 200 \AA$ column (The Nest Group Inc.; Southborough, MA, USA) with buffers as follows. Mobile phase A was $5 \mathrm{mM}$ monopotassium phosphate $(25 \% \mathrm{v} / \mathrm{v}$ acetonitrile, $\mathrm{pH} 3.0)$, and mobile phase $\mathrm{B}$ was $5 \mathrm{mM}$ phosphate and $350 \mathrm{mM}$ potassium chloride $(25 \%$ $\mathrm{v} / \mathrm{v}$ acetonitrile, $\mathrm{pH}$ 3.0). Dried iTRAQ-labeled samples were resuspended in $300 \mu \mathrm{L}$ of mobile phase A and injected onto the SCX column. The gradient for SCX was $0-3 \mathrm{~min}, 0 \% \mathrm{~B}$; 3-45 $\mathrm{min}, 0-75 \% \mathrm{~B}$; 45-50 $\mathrm{min}, 75-100 \% \mathrm{~B}$; 50-55 $\mathrm{min}, 100 \%$ mobile phase B; $55-56 \mathrm{~min}, 100-0 \% \mathrm{~B}$; and $56-106 \mathrm{~min}, 0 \%$ B. Thirteen SCX fractions were collected, and each fraction was desalted with an HLB cartridge.

2.5. $L C-M S / M S$. Online desalting and reversed phase chromatography was performed with a Nano 2D-LC system equipped with an autosampler (Eksigent; Dublin, CA, USA). Mobile phase A and B for these analyses were 3\% (v/v) acetonitrile with $0.1 \%$ formic acid and $100 \%(\mathrm{v} / \mathrm{v})$ acetonitrile with $0.1 \%$ formic acid, respectively. SCX fractions were solubilized in $50 \mu \mathrm{L}$ of $\mathrm{H}_{2} \mathrm{O}$ with $0.1 \%$ formic acid 
and filtered with a $0.45 \mu \mathrm{m}$ filter (Thermo Fisher Scientific; Waltham, MA, USA). For each run, $5 \mu \mathrm{L}$ of sample was loaded into a trapping column $(100 \mu \mathrm{m}$ i.d. $\times 2 \mathrm{~cm})$, which was packed in-house with $\mathrm{C}_{18} 200 \AA$ stationary phase material (Michrom Bioresource Inc.; Auburn, CA, USA) at $3 \mu \mathrm{L} \cdot \mathrm{min}^{-1}$ in $3 \%$ mobile phase B for $3 \mathrm{~min}$. After desalting, the sample was loaded into the analytical column $(75 \mu \mathrm{m}$ i.d. $\times 13.2 \mathrm{~cm})$, which was packed in-house with $\mathrm{C}_{18} 100 \AA$ stationary phase material (Michrom Bioresource Inc.). The gradient was as follows: $0-5 \mathrm{~min}, 10 \%$ mobile phase $\mathrm{B} ; 5-75 \mathrm{~min}, 10-$ $30 \%$ B; 75-95 min, 30-60\% B; 95-100 min, 60-90\% B; 100$105 \mathrm{~min}, 90-10 \% \mathrm{~B}$; and $110-120 \mathrm{~min}, 10 \% \mathrm{~B}$. The LC eluent was analyzed with positive-ion nanoflow electrospray using a LTQ-Orbitrap Velos mass spectrometer (Thermo-Fisher Scientific, Waltham, MA, USA). Data-dependent acquisition parameters were as follows: the MS survey scan in the Orbitrap was 60,000 resolution over $300-1800 \mathrm{~m} / z$; CID was performed on the ion trap with normalized collision energy $35 \%$; HCD was recorded in the Orbitrap with normalized collision energy $45 \%$ and 7,500 resolution; the top six most intense ions in the parent MS scan were selected and activated using CID and HCD [24]; dynamic exclusion was enabled with a repeat count of 2 for a duration of $60 \mathrm{sec}$; a minimum of 5000 ion counts were necessary for fragmentation events. Each fraction was subject to triplicate LC-MS/MS.

2.6. Database Searching. RAW files were analyzed with Proteome Discoverer 1.2 software (Thermo). Both CID and HCD spectra were used to obtain sequence information against the UniProt human database (04/25/2010, 20295 sequences). Sequest search parameters were as follows: enzyme specificity was trypsin with two maximum miscleavages; precursor mass tolerance was $10 \mathrm{ppm}$; fragment mass tolerance was $0.8 \mathrm{Da}$; N-terminus and lysine modification with iTRAQ $(144.102 \mathrm{Da})$ and cysteine carbamidomethylation $(57.021 \mathrm{Da})$ were set as fixed modifications; tyrosine modification with iTRAQ was set as a dynamic modification. Decoy database searching was employed to generate medium $(P<0.05)$ and high $(P<0.01)$ confidence peptide lists. All peptides with medium and high confidence were pooled into a single data file and used for final protein identification and quantitation. Proteins with at least two spectral counts in a workflow replicate were included for identification. Only proteins with at least two spectral counts in a technical replicate were considered for quantitative and statistical analysis. Oneway ANOVA analysis $(P<0.05)$ was performed for proteins quantified in at least two workflow replicates utilizing Microsoft Excel.

2.7. Protein Quantification and Statistical Analysis. Peptide ratios (e.g., 115/114, 116/114, and 117/114) were calculated based on the peak intensity of each reporter ion. The protein ratios were the median ratio of the corresponding peptide ratios. Coefficients of variation (CV) values were calculated for ratios of proteins quantified in at least two workflow replicates. The mean $\mathrm{CV}$ value across workflow replicates was calculated and used as the total biological variation, $S_{b}$. The technical variation, $S_{t}$, was calculated for proteins quantified in at least two LC-MS/MS analyses within an individual workflow. The relation between the fold change $(F)$, random variation $(S)$, biological replicates per group $(n)$, and technical replicates $(m)$ has been previously reported [25] and is expressed by the formula

$$
\begin{gathered}
n=\frac{2(Z+T)^{2} S^{2}}{(F-1)^{2}}, \\
S=\left(\frac{S_{b}^{2}+S_{t}^{2}}{m}\right)^{1 / 2} .
\end{gathered}
$$

The quantities $Z$ and $T$ depend on the power of the test and the significance level, respectively. The power and significance levels were set as 0.8 and 0.05 , respectively, such that the formula approximates to

$$
\begin{gathered}
n=\frac{20 S^{2}}{(F-1)^{2}}, \\
F=\frac{4.47 S}{n^{1 / 2}}+1 .
\end{gathered}
$$

One-way ANOVA analysis $(P<0.05)$ was performed for proteins quantified in at least two workflow replicates utilizing Microsoft Excel.

\section{Results and Discussion}

A robust quantitative shotgun proteomics workflow (Figure 1(a)) was assessed for its ability to identify new human plasma proteins and to guide future experimental designs. The workflow uses tandem MARS depletion (TMD), iTRAQ four-plex reagents, SCX fractionation, and nanoflow LC-MS/MS on a LTQ-Orbitrap Velos MS. The entire workflow was repeated three times using new aliquots of four plasma samples that were subject to TMD using a Hu 6 MARS column. The time it takes to complete a single workflow replicate is $\sim 7$ days with a majority of the costs being attributed to the MARS column ( 200 analyses per column) and the iTRAQ reagents (5 analyses per kit). Immunodepletion of samples is very reproducible for single-stage MARS depletion (MD, Figure 1(b)) and TMD (Figure 1(c)). It is apparent from the chromatograms (Figures 1(b) and 1(c)) that high abundance proteins (i.e., $\left.t_{r} \sim 12.5 \mathrm{~min}\right)$ are substantially depleted after the TMD step. The average $\%$ depletion of the six high abundance proteins is $88 \%$ and $92 \%$ for MD and TMD, respectively, (see Supplementary Table S1 of the Supplementary Material available online at http://dx.doi.org/10.1155/2013/654356) and is similar to that obtained using other tandem depletion strategies $[6,8,9]$. It should be noted that albumin was still detectable after TMD (Supplementary Table S2); however other abundant proteins (i.e., $\alpha$-1-antitrypsin, IgG, IgA, transferrin, and haptoglobin) did not have any observed peptide hits. The most abundant protein detected based on spectral counts was complement C3 which had an average total spectral count (SC) of $>4000$ across the workflow replicates (Supplementary Table S2). The use of a single column to perform dual 


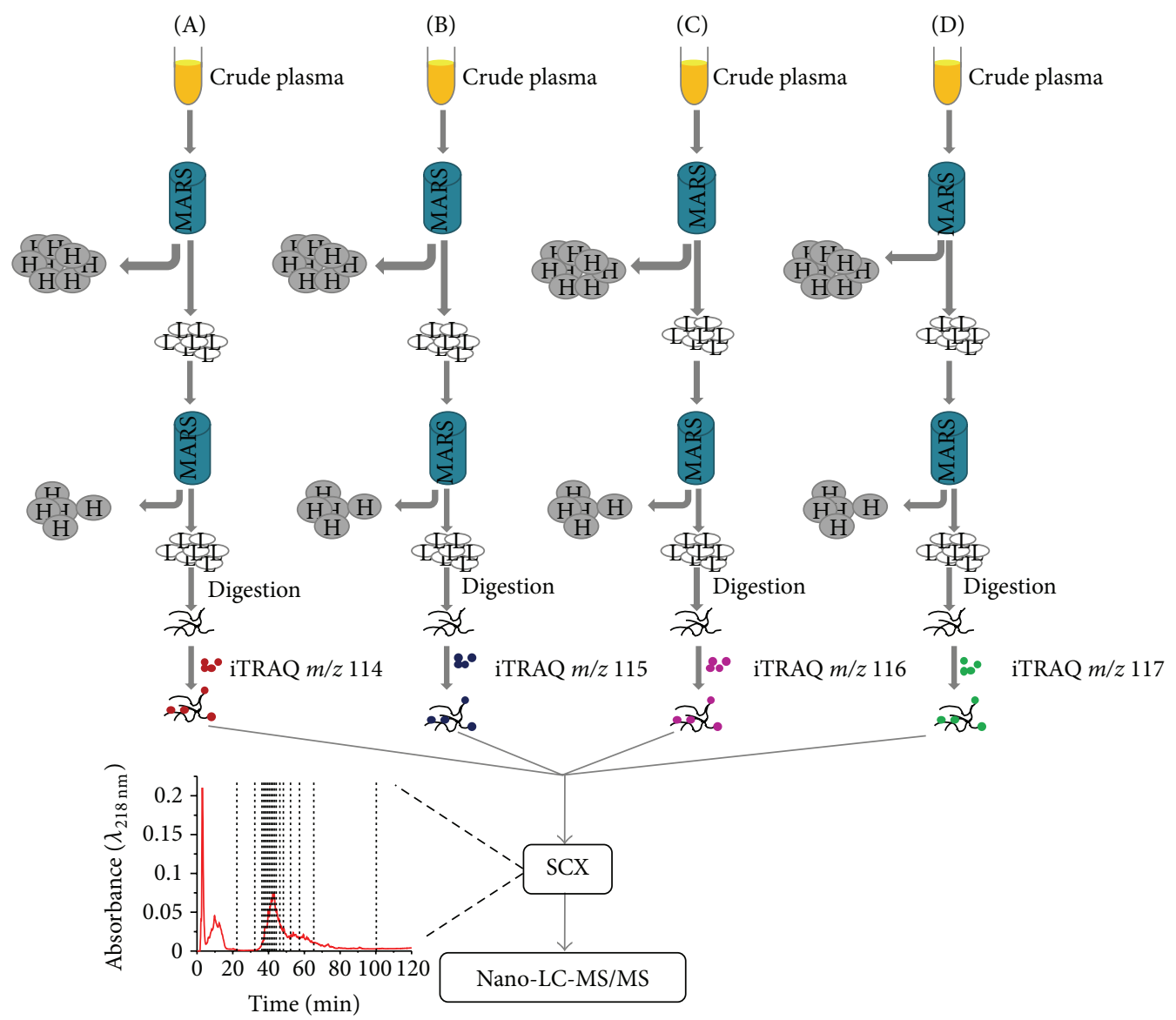

(a)

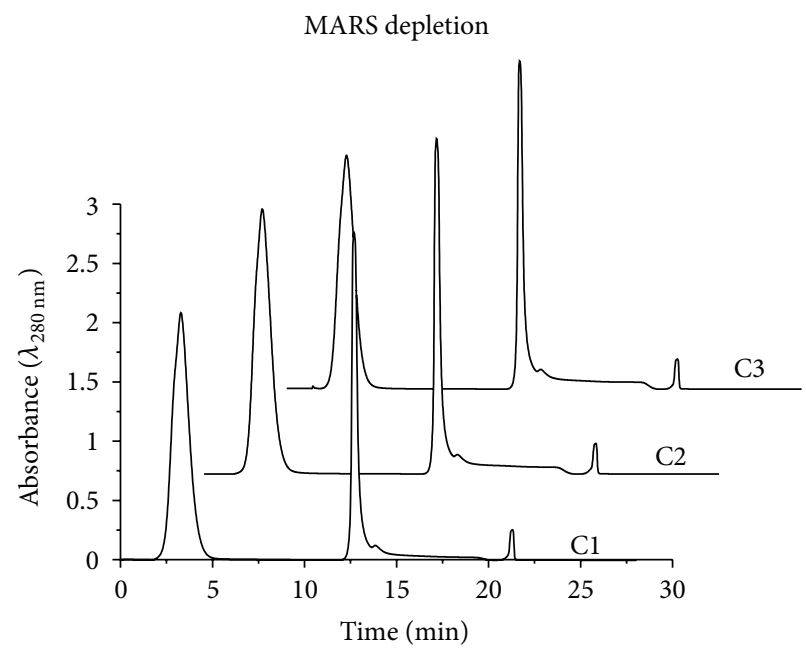

(b)

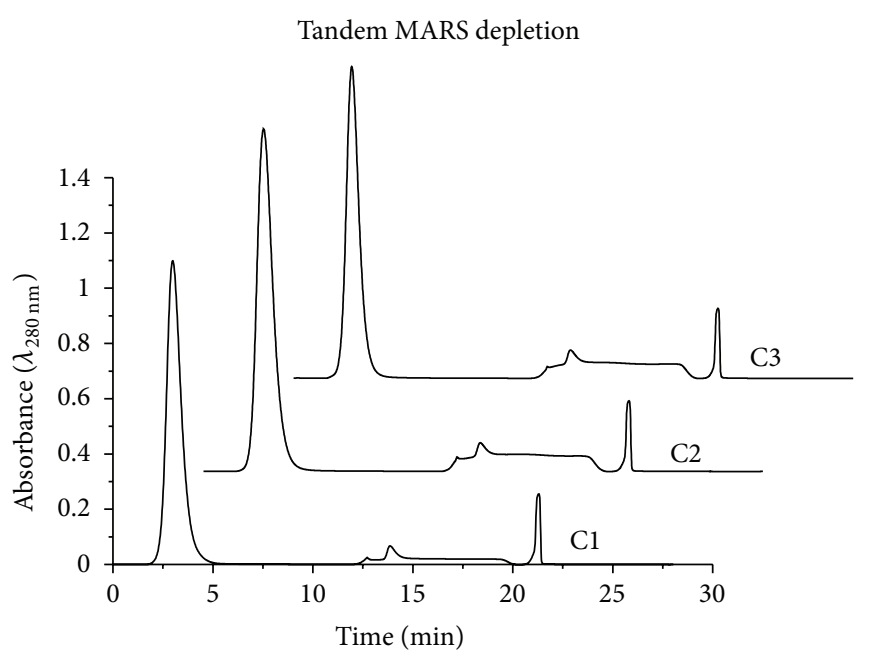

(c)

FIGURE 1: (a) The iTRAQ-based quantitative platform used for plasma proteome analyses in which the flow-through fractions from four crude plasma samples (A-D) are modified with iTRAQ 4-plex reagents, pooled into a single mixture, and separated with offline SCX-LC-MS/MS. Example chromatograms $\left(\lambda_{280 \mathrm{~nm}}\right)$ from three independent injections of plasma sample C upon (b) MD and (c) TMD are shown.

immunodepletion minimizes the expenses associated with the use of multiple MARS or other depletion columns.

TMD samples were used for further iTRAQ tagging reactions and analyzed with SCX LC-MS/MS (Figure 1(a)). A total of 689 unique proteins were identified from the combined results of the three independent workflow experiments (Supplementary Table S2) and are slightly larger than the number of proteins observed in other reports $[5,9,19,26,27]$. 


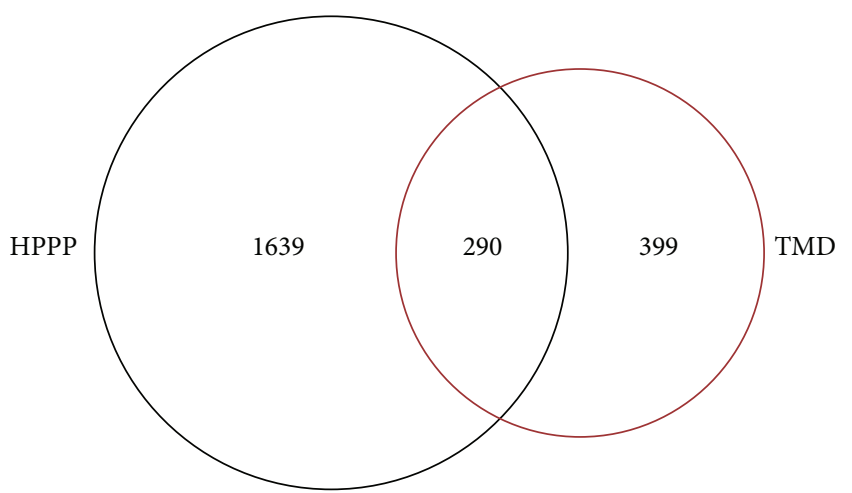

(a)

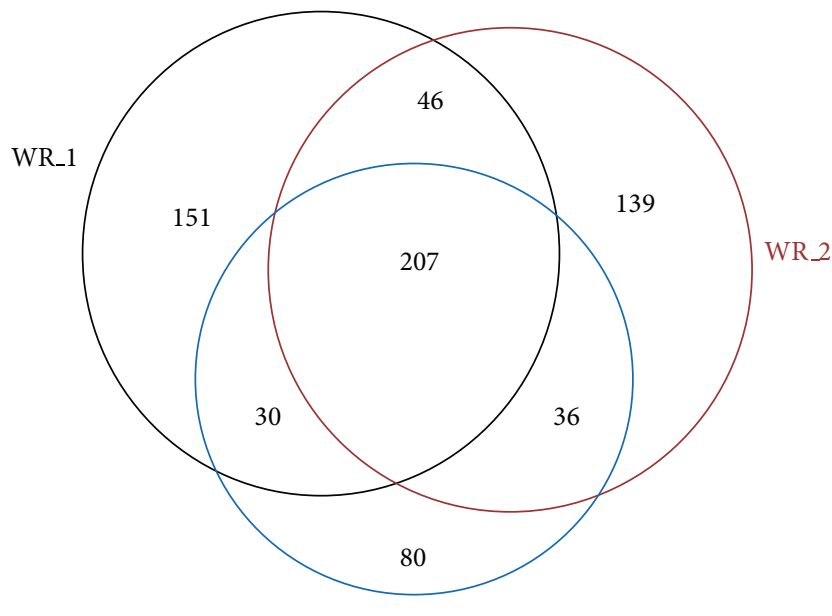

WR_3

(b)

FIgURE 2: (a) Venn diagram for proteins identified in the human plasma proteome project (HPPP) and the TMD workflow presented herein. (b) Venn diagram for proteins identified in three workflow replicate (WR) experiments.

The proteins identified in this study were compared to the recently released 2011 HUPO plasma protein database to assess the depth of proteome coverage. Based on comparisons of identified proteins to the 1929 nonredundant sequences reported in the Human Proteome Organization Database [10], 399 novel proteins with $\geq 2$ SC are uniquely observed in these studies (Figure 2(a)). Although the incorporation of a dual depletion step and SCX fractionation increases experimental sample preparation time, our results support the necessity of these (or similar) steps for identification of commonly detected and novel plasma proteins. Due to different experimental designs, LC-MS/MS data acquisition settings, and searching engines, the number of identified proteins may vary a lot across different labs. It is also possible that a portion of the identifications are a result of profiles specific to the patient samples employed. All of the proteins identified are provided in Supplementary Table S2. A total of 207 proteins were observed in all three of the workflow experiments, and more than half of the total proteins were observed in a single workflow replicate (Figure 2(b)). With more stringent criteria (i.e., not less than 2 unique peptides for protein identification), 229 proteins were identified across three workflow replicates, and 40 new proteins were identified in these studies in comparison to the HUPO database.

The datasets collected from this TMD strategy were used to examine the variation in the entire workflow. iTRAQ reporter ion (i.e., $m / z 115,116$, and 117) ratios were calculated with respect to $m / z 114$ for each protein. Proteins quantified by at least 2 spectral counts were used in the assessment of variation. Of the 207 proteins identified in all three workflow replicates, 139 proteins (with at least 2 spectral counts) were quantified in the Proteome Discoverer Analysis. These proteins were used to initially assess the variance in reporter ion ratios across the workflow replicates (of which each includes three technical replicates) by employing wellestablished statistical approaches [25, 28-31]. We refer to a technical replicate as the cumulative results obtained across individual LC-MS/MS analyses of the 13 SCX fractions. Thus within a single workflow experiment three technical replicates were measured. The workflow replicate assesses the variation beginning with the start of the plasma sample preparation.

Figure 3(a) plots the distribution of CV values for proteins as a function of reporter ion ratios (e.g., 115/114, 116/114, and 117/114). The distribution of SD values for proteins as a function of $\log _{2}$ transformed ratios are provided in Supplementary Figure 1. Within a single workflow replicate, the average reporter ion ratio across technical replicates was calculated for individual proteins. The corresponding mean (and median) CV values for ratios 115/114, 116/114, and 117/114 across all proteins quantified in the three workflow replicates was $\pm 0.16(0.13), 0.13(0.11)$, and $0.11(0.09)$, respectively. Seventy-five percent of proteins had a CV $<0.16$, and $90 \%$ of proteins had a $\mathrm{CV}<0.21$ when reporter ion 114 was used as the reference channel. Because the reporter ion channel used as the reference can have some effect on quantitation [19], the mean (and median) CV values were also calculated for different reference channels (Supplementary Table S3). When reporter ions $m / z 115,116$, and 117 were used as the reference channel, $90 \%$ of proteins had a $\mathrm{CV}$ value $<0.28,0.21$, and 0.24 , respectively. This range of $\mathrm{CV}$ values that results from selection of different reference channels reflects the variation inherent in the four plasma samples as well as any variation that arises during LC-MS/MS analysis.

Incorporation of multiple workflow or technical replicates does not imply that proteins will be observed in all experiments (Figure 2(a)); therefore CV values were also calculated for the 71 proteins that were only quantified in any two of the three workflow replicates. When reporter ion $\mathrm{m} / z 114$ was used as the reference channel, the mean (and median) CV was $\pm 0.30(0.23), 0.20(0.15)$, and $0.18(0.15)$ for ratios 115/114, 116/114, and 117/114, respectively (Supplementary Table S3). The higher CV observed for this set of proteins agrees with the notion that less replication (workflow and technical) could lead to higher variation in reporter ion ratios $[20,28]$ as well as biases that arise in low abundance proteins due to lower numbers of detected spectral counts and higher variability due to lower intensity signals [22]. Higher variability in reporter ion ratios correlated with 


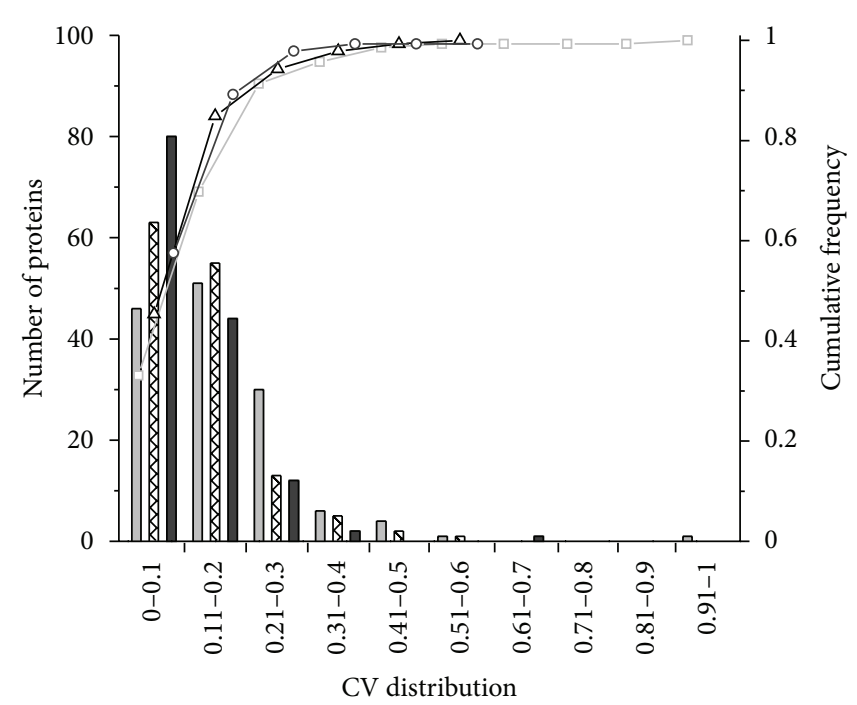

[ Number of proteins for ratio $I_{115} / I_{114}$

- $\square$ - Cumulative frequency for ratio $I_{115} / I_{114}$

1 Number of proteins for ratio $I_{116} / I_{114}$

$-\Delta-$ Cumulative frequency for ratio $I_{116} / I_{114}$

- Number of proteins for ratio $I_{117} / I_{114}$

- - Cumulative frequency for ratio $I_{117} / I_{114}$

(a)

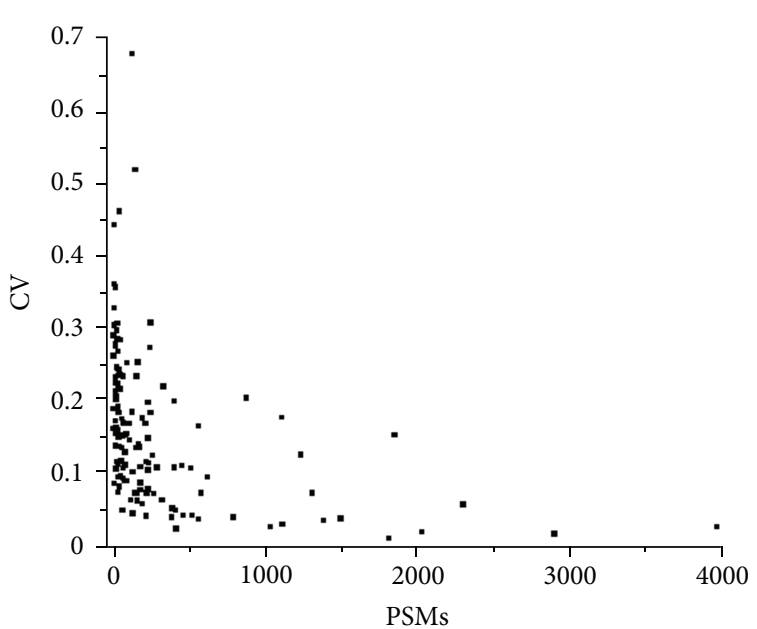

(b)

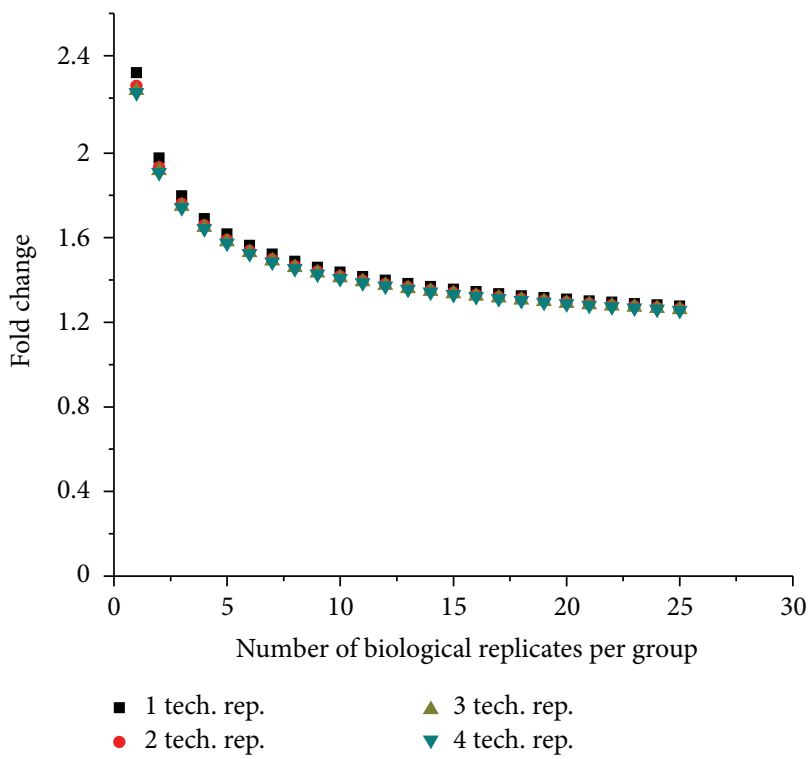

(c)

FIgure 3: (a) The distribution of CV values for $I_{115} / I_{114}$ (grey rectangular), $I_{116} / I_{114}$ (shaded rectangular), and $I_{117} / I_{114}$ (black rectangular) for proteins quantified in each of the three independent experiments $(N=139$ proteins). The cumulative frequency of proteins with specific $\mathrm{CV}$ values for $I_{115} / I_{114}$ (dashed square), $I_{116} / I_{114}$ (dashed triangle), and $I_{117} / I_{114}$ (dashed circle) are shown. CV values are given as fractional values. The total peak intensity is represented by $I$. (b) Plot of the mean CV values for reporter ion ratios relative to reference channel 114 as a function of the number of spectral counts identified for each protein. Only proteins identified in all three workflow replicates are represented in this plot. (c) Power analysis for iTRAQ-based quantitative platform whereby fold change values are plotted for a given number of biological replicates as a function of the number of technical replicates (i.e., $m=1$ to 4 ). The power and significance level values were set to $80 \%$ and 0.05 , respectively. 
proteins that were identified with lower numbers of spectral counts (Figure 3(b)).

In order to estimate the overall variance of this workflow, $\mathrm{CV}$ values were obtained for proteins quantified in at least two of the workflow replicates $(N=210)$. The mean $C V$ was 0.21 , 0.15 , and 0.13 for ratios $115 / 114,116 / 114$, and $117 / 114$, respectively, and similar values were obtained for other reference channels (Supplementary Table S3). Taking the CV values of reference channel 114 into consideration, the overall variation in the entire plasma workflow is $\sim 0.16$. Herein, the technical variation was assessed by considering proteins observed in multiple LC-MS/MS analyses for individual workflow replicates. The technical variation is $\sim 0.10$ for proteins quantified in at least two replicates (Supplementary Table S4). In order to determine proteins that were quantified similarly across workflow replicates, one-way ANOVA analysis $(P<0.05)$ [31] was carried out. Based on these results, $\sim 70 \%$ of the 210 quantified proteins have similar ratios across workflow replicates (data not shown).

Power analysis was also performed in order to assess the fold-change criterion that should be applied based on a given number of biological replicates (Figure 3(c)). We note that our experimental approach (i.e., repeating the workflow using new aliquots of the same plasma samples) does not represent a true biological replicate. However, this analysis still provides statistical insight to the power of biological replication in future experimental designs. The total biological variance $\left(S_{b}\right)$, technical variance $\left(S_{t}\right)$, power, and significance level applied were $0.16,0.10,80 \%$, and 0.05 , respectively. As indicated in Figure 3(c), if ten biological replicates per group are used then a fold-change cutoff of 1.3 can be applied, and only two replicates are required to use the commonly applied 2.0 fold-change cutoff. Technical replicates do not appear to have a significant effect on the fold-change criterion when multiple biological replicates will be used (Figure 3(c)). These data provide additional evidence to support the notion that biological replication (i.e., in these studies workflow replication) is one of the most important factors that should be considered in the experimental design $[16,21,25]$.

This paper has presented a robust quantitative plasma proteomics workflow that involves tandem MARS depletion, iTRAQ tagging, and SCX-LC-MS/MS analysis. The use of TMD and SCX fractionation resulted in the identification of 689 proteins with $\geq 2$ SC. Compared to the HUPO database, $\sim 400$ of these proteins were previously unreported. The use of TMD and SCX fractionation significantly increases the number of proteins detected. The overall variation in the presented workflow ranges from \pm 11 to $30 \%$, and power analysis indicates that increasing biological replication would allow a lower fold-change cutoff to be applied to determine statistically relevant differentially expressed proteins. Future studies from our laboratory involve the application of this workflow to specific disease states whereby biological replicates are also being incorporated into the experimental design.

\section{Conflict of Interests}

The authors have declared no conflict of interests.

\section{Acknowledgments}

The authors acknowledge the University of Pittsburgh and the Society of Analytical Chemists in Pittsburgh for funds to support this work and the CRISMA laboratory for plasma samples. GenIMS was funded by the National Institute of General Medical Sciences R01 GM61992 Grant with additional support from GlaxoSmithKline for enrollment and clinical data collection.

\section{References}

[1] N. L. Anderson and N. G. Anderson, “The human plasma proteome: history, character, and diagnostic prospects," Molecular \& Cellular Proteomics, vol. 1, no. 11, pp. 845-867, 2002.

[2] Y. Gong, X. Li, B. Yang et al., "Different immunoaffinity fractionation strategies to characterize the human plasma proteome," Journal of Proteome Research, vol. 5, no. 6, pp. 1379-1387, 2006.

[3] H. J. Issaq, Z. Xiao, and T. D. Veenstra, "Serum and plasma proteomics," Chemical Reviews, vol. 107, no. 8, pp. 3601-3620, 2007.

[4] J. M. Jacobs, J. N. Adkins, W. J. Qian et al., "Utilizing human blood plasma for proteomic biomarker discovery," Journal of Proteome Research, vol. 4, no. 4, pp. 1073-1085, 2005.

[5] C. Tu, P. A. Rudnick, M. Y. Martinez et al., "Depletion of abundant plasma proteins and limitations of plasma proteomics," Journal of Proteome Research, vol. 9, no. 10, pp. 4982-4991, 2010.

[6] P. Juhasz, M. Lynch, M. Sethuraman et al., "Semi-targeted plasma proteomics discovery workflow utilizing two-stage protein depletion and off-line LC-MALDI MS/MS," Journal of Proteome Research, vol. 10, no. 1, pp. 34-45, 2011.

[7] N. Mortezai, S. N. Harder, C. Schnabel et al., "Tandem affinity depletion: a combination of affinity fractionation and immunoaffinity depletion allows the detection of low-abundance components in the complex proteomes of body fluids," Journal of Proteome Research, vol. 9, no. 12, pp. 6126-6134, 2010.

[8] W.-J. Qian, D. T. Kaleta, B. A. Ogata et al., "Enhanced detection of low abundant human plasma proteins using a tandem IgY12SuperMix immunoaffinity separation strategy," Molecular \& Cellular Proteomics, vol. 7, no. 10, pp. 1963-1973, 2008.

[9] C. M. Shuford, A. M. Hawkridge, J. C. Burnett, and D. C. Muddiman, "Utilizing spectral counting to quantitatively characterize tandem removal of abundant proteins (TRAP) in human plasma," Analytical Chemistry, vol. 82, no. 24, pp. 1017910185, 2010.

[10] T. Farrah, E. W. Deutsch, G. S. Omenn et al., "A high-confidence human plasma proteome reference set with estimated concentrations in PeptideAtlas," Molecular \& Cellular Proteomics, vol. 10, Article ID M110.006353, 2011.

[11] C. D. Aluise, R. A. Sowell, and D. A. Butterfield, "Peptides and proteins in plasma and cerebrospinal fluid as biomarkers for the prediction, diagnosis, and monitoring of therapeutic efficacy of Alzheimer's disease," Biochimica et Biophysica Acta, vol. 1782, no. 10, pp. 549-558, 2008.

[12] E. Boja, T. Hiltke, R. Rivers et al., "Evolution of clinical proteomics and its role in medicine," Journal of Proteome Research, vol. 10, no. 1, pp. 66-84, 2011.

[13] D. Han, S. Moon, H. Kim et al., "Detection of differential proteomes associated with the development of type 2 diabetes in the Zucker rat model using the iTRAQ technique," Journal of Proteome Research, vol. 10, no. 2, pp. 564-577, 2011. 
[14] M. Latterich, M. Abramovitz, and B. Leyland-Jones, "Proteomics: new technologies and clinical applications," European Journal of Cancer, vol. 44, no. 18, pp. 2737-2741, 2008.

[15] N. A. Karp, W. Huber, P. G. Sadowski, P. D. Charles, S. V. Hester, and K. S. Lilley, "Addressing accuracy and precision issues in iTRAQ quantitation," Molecular and Cellular Proteomics, vol. 9 , no. 9, pp. 1885-1897, 2010.

[16] S. G. Chee, K. C. Poh, K. P. Trong, and P. C. Wright, "Technical, experimental, and biological variations in isobaric tags for relative and absolute quantitation (iTRAQ)," Journal of Proteome Research, vol. 6, no. 2, pp. 821-827, 2007.

[17] E. G. Hill, J. H. Schwacke, S. Comte-Walters et al., "A statistical model for iTRAQ data analysis," Journal of Proteome Research, vol. 7, no. 8, pp. 3091-3101.

[18] A. L. Oberg, D. W. Mahoney, J. E. Eckel-Passow et al., "Statistical analysis of relative labeled mass spectrometry data from complex samples using ANOVA," Journal of Proteome Research, vol. 7, no. 1, pp. 225-233, 2008.

[19] X. Song, J. Bandow, J. Sherman et al., "iTRAQ experimental design for plasma biomarker discovery," Journal of Proteome Research, vol. 7, no. 7, pp. 2952-2958, 2008.

[20] P. K. Chong, C. S. Gan, T. K. Pham, and P. C. Wright, "Isobaric tags for relative and absolute quantitation (iTRAQ) reproducibility: implication of multiple injections," Journal of Proteome Research, vol. 5, no. 5, pp. 1232-1240, 2006.

[21] C. Zhou, K. L. Simpson, L. J. Lancashire et al., "Statistical considerations of optimal study design for human plasma proteomics and biomarker discovery," Journal of Proteome Research, vol. 11, no. 4, pp. 2103-2113, 2012.

[22] D. W. Mahoney, T. M. Therneau, C. J. Heppelmann et al., "Relative quantification: characterization of bias, variability and fold changes in mass spectrometry data from iTRAQ-labeled peptides," Journal of Proteome Research, vol. 10, no. 9, pp. 43254333, 2011.

[23] J. A. Kellum, L. Kong, M. P. Fink et al., "Understanding the inflammatory cytokine response in pneumonia and sepsis: results of the genetic and inflammatory markers of sepsis (GenIMS) study," Archives of Internal Medicine, vol. 167, no. 15, pp. 1655-1663, 2007.

[24] T. Köcher, P. Pichler, M. Schutzbier et al., "High precision quantitative proteomics using iTRAQ on an LTQ Orbitrap: a new mass spectrometric method combining the benefits of all," Journal of Proteome Research, vol. 8, no. 10, pp. 4743-4752, 2009.

[25] G. W. Horgan, "Sample size and replication in 2D gel electrophoresis studies," Journal of Proteome Research, vol. 6, no. 7, pp. 2884-2887, 2007.

[26] E. Ernoult, A. Bourreau, E. Gamelin, and C. Guette, "A proteomic approach for plasma biomarker discovery with iTRAQ labelling and OFFGEL fractionation," Journal of Biomedicine \& Biotechnology, vol. 2010, p. 927917, 2010.

[27] H. Ye, L. Sun, X. Huang, P. Zhang, and X. Zhao, "A proteomic approach for plasma biomarker discovery with 8-plex iTRAQ labeling and SCX-LC-MS/MS," Molecular and Cellular Biochemistry, vol. 343, no. 1-2, pp. 91-99, 2010.

[28] D. A. ) Cairns, "Statistical issues in quality control of proteomic analyses: good experimental design and planning," Proteomics, vol. 11, no. 6, pp. 1037-1048.

[29] N. A. Karp and K. S. Lilley, "Design and analysis issues in quantitative proteomics studies," Proteomics, vol. 7, pp. 42-50, 2007.

[30] Y. Levin, "The role of statistical power analysis in quantitative proteomics," Proteomics, vol. 11, no. 12, pp. 2565-2567, 2011.
[31] A. L. Oberg and O. Vitek, "Statistical design of quantitative mass spectrometry-based proteomic experiments," Journal of Proteome Research, vol. 8, no. 5, pp. 2144-2156, 2009. 

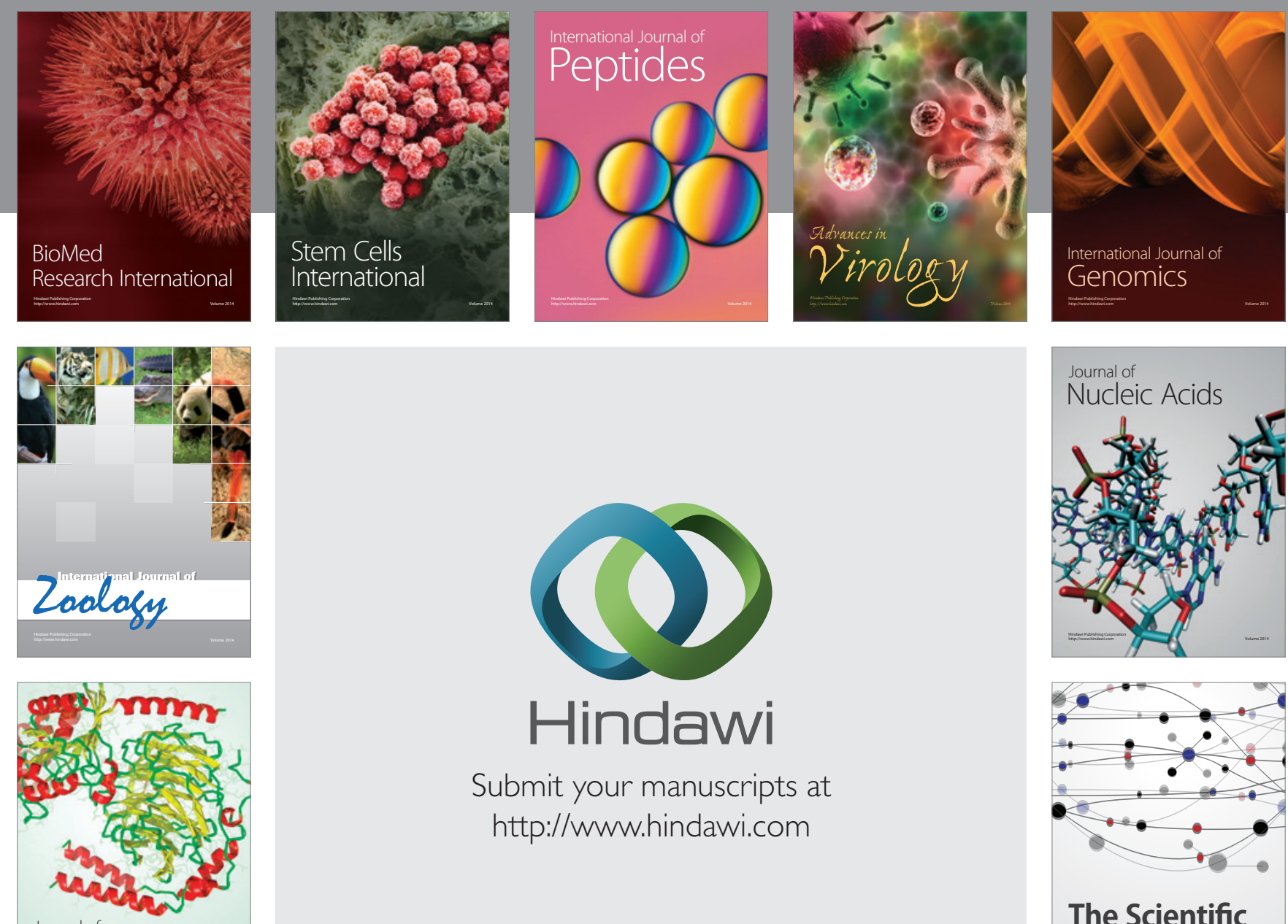

Submit your manuscripts at

http://www.hindawi.com

Journal of
Signal Transduction
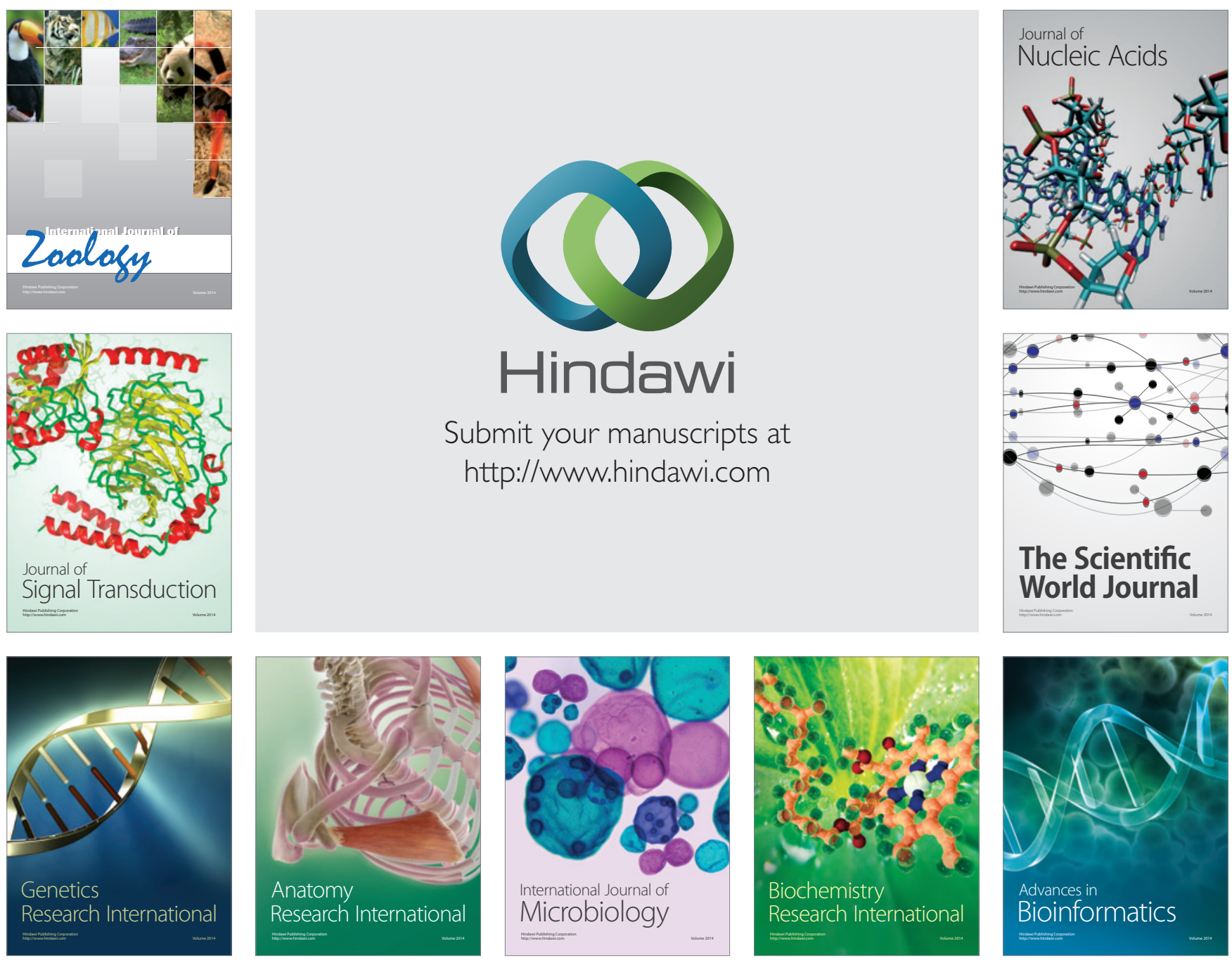

The Scientific World Journal
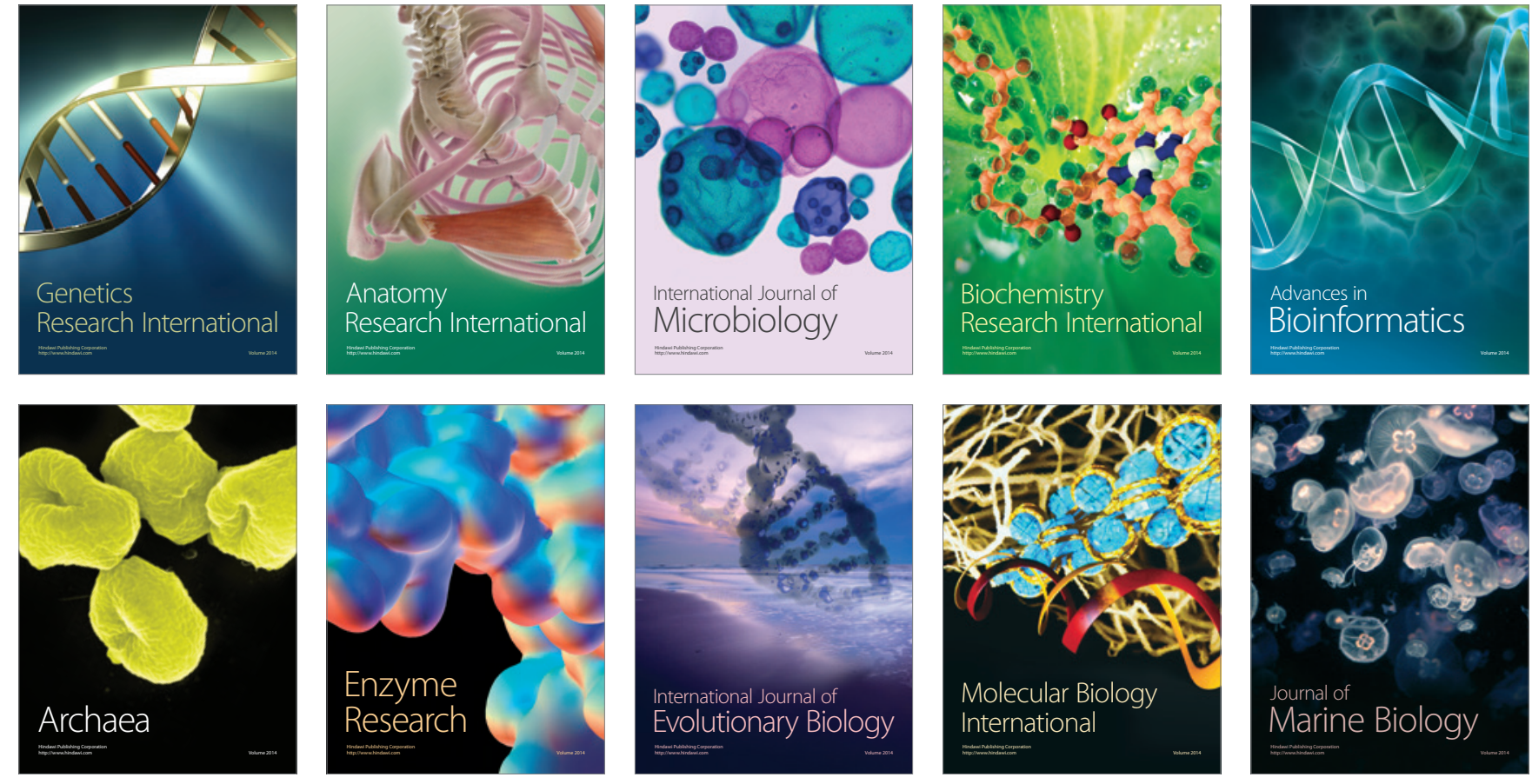